\title{
Effect of therapeutic plasma exchange on endothelial activation and coagulation- related parameters in septic shock
}

Klaus Stahl'10, Julius J. Schmidt ${ }^{2}$, Benjamin Seeliger ${ }^{3}$, Bernhard M. W. Schmidt ${ }^{2}$, Tobias Welte ${ }^{3}$, Hermann Haller ${ }^{2}$, Marius M. Hoeper ${ }^{3}$ Ulrich Budde ${ }^{4}$, Christian Bode ${ }^{5}$ and Sascha David ${ }^{2 *}$

\begin{abstract}
Background: A dysbalanced coagulation system is part of the pathological host response to infection in sepsis. Activation of pro-coagulant pathways and attenuation of anti-coagulant activity ultimately lead to microvascular stasis and consequent organ failure. No treatment approaches specifically targeting this axis are available. We explored the effects of therapeutic plasma exchange (TPE) on microvascular coagulation dysbalance in septic shock.

Methods: We conducted a prospective single-center study enrolling 31 patients with early septic shock (onset $<12$ h) requiring high doses of norepinephrine (NE $>0.4 \mu \mathrm{g} / \mathrm{kg} / \mathrm{min}$ ). Clinical and biochemical data, including measurement of protein $C_{\text {; }}$ a disintegrin and metalloprotease with a thrombospondin type 1 motif, member 13 (ADAMTS13); and von Willebrand factor antigen (VWF:Ag), were obtained before and after TPE against fresh frozen plasma.

Results: Antithrombotic acting proteins such as antithrombin-III (ATIII) and protein C were markedly reduced in septic patients, but their activity increased after TPE (ATIII, 51\% (41-61) vs. 63\% (48-70), $p=0.029$; protein C, 47\% (38-60) vs. 62\% (54-69), $p=0.029)$. Median ADAMTS13 activity was increased by TPE from $27(21-42) \%$ before to 47 (38-62) \% after TPE $(p<0.001)$. In contrast, vWF:Ag was elevated and could be reduced by TPE (353 (206-492) $\mathrm{IU} / \mathrm{dL}$ vs. 170 (117-232) IU/dL, $p<0.001)$. Regression analysis yielded a correlation between ADAMTS13 activity and platelet count $\left(p=0.001, R^{2}=0.316\right)$.
\end{abstract}

Conclusions: Septic shock was associated with activation of pro-coagulant pathways and simultaneous depletion of anti-coagulant factors. TPE partially attenuated this dysbalance by removing pro- and by replacing anti-coagulant factors.

Trial registration: ClinicalTrials.gov, NCT03065751. Retrospectively registered on 28 February 2017.

Keywords: Extracorporeal treatment, Septic shock, Plasmapheresis, ADAMTS-13, von Willebrand factor

\footnotetext{
* Correspondence: david.sascha@mh-hannover.de

${ }^{2}$ Department of Nephrology and Hypertension, Hannover Medical School, Carl-Neuberg-Str.1, 30625 Hannover, Germany

Full list of author information is available at the end of the article
}

C C The Author(s). 2020 Open Access This article is licensed under a Creative Commons Attribution 4.0 International License, which permits use, sharing, adaptation, distribution and reproduction in any medium or format, as long as you give appropriate credit to the original author(s) and the source, provide a link to the Creative Commons licence, and indicate if changes were made. The images or other third party material in this article are included in the article's Creative Commons licence, unless indicated otherwise in a credit line to the material. If material is not included in the article's Creative Commons licence and your intended use is not permitted by statutory regulation or exceeds the permitted use, you will need to obtain permission directly from the copyright holder. To view a copy of this licence, visit http://creativecommons.org/licenses/by/4.0/ The Creative Commons Public Domain Dedication waiver (http://creativecommons.org/publicdomain/zero/1.0/) applies to the data made available in this article, unless otherwise stated in a credit line to the data. 


\section{Background}

Sepsis is defined as a life-threatening organ dysfunction caused by a dysregulated host response to infection; if hypotension is refractory to volume resuscitation and serum lactate is elevated, it is termed septic shock [1]. In the absence of a specific intervention other than antiinfective drugs, mortality associated with septic shock is still as high as $60 \%$ [2]. The overwhelming host response consisting of cytokine release, attraction of inflammatory cells, and global endothelial activation is the key driver of morbidity and mortality [3]. Endothelial dysfunction leads to systemic aggregation of platelets in virtually all microvascular beds with consecutive consumption of coagulation factors. Clinically, this situation is well known as disseminated intravascular coagulation (DIC) resulting in widespread clotting of the microvasculature system and progressive multi-organ failure [4].

The critical role of acquired protein $\mathrm{C}$ deficiency in septic shock with purpura fulminans has been recognized for a long time [5-8], and some experts suggest to supplement protein $\mathrm{C}$ if its activity is severely lowered [8]. Interestingly, the only ever approved "specific sepsis therapeuticum" was an activated protein $C$ preparation $\left(\right.$ Xigris $\left.^{\odot}\right)$ [9], before it was later removed from the market due to a large-scale negative follow-up study [10].

A disintegrin and metalloprotease with a thrombospondin type 1 motif, member 13 (ADAMTS13) is the most important modulator of size and function of the von Willebrand factor antigen (vWF:Ag) in human plasma by cleaving preformed vWF multimer chains [11]. Severe ADAMTS13 deficiency causes accumulation of ultra-large vWF multimers (ULVWF) leading to the clinical picture of severe thrombotic microangiopathy as seen in thrombotic thrombocytopenic purpura (TTP) [12]. More recently, it has been demonstrated that a significant deficiency of ADAMTS13 also appears in sepsis [13-15]. At the same time, large amounts of vWF:Ag are secreted by the activated septic endothelium leading to both increased platelet aggregation and formation of highly pro-thrombotic ULVWF [16]. In fact, an increased vWF:Ag/ADAMTS13 ratio has been repeatedly associated with the severity of shock and organ failure as well as increased mortality in sepsis [13, 17-20].

Our group has recently described the use of therapeutic plasma exchange (TPE) against fresh frozen plasma (FFP) as an adjunctive treatment strategy in early (onset $<12 \mathrm{~h}$ ) and severe (norepinephrine (NE) dose $>0.4 \mu \mathrm{g} / \mathrm{kg} / \mathrm{min}$ ) septic shock [21]. Although uncontrolled, we observed rapid stabilization of hemodynamics, improvement of fluid balances, and reduction of key pro-inflammatory cytokines and permeability factors. We believe that these positive surrogate effects can be attributed to two important aspects: (1) removal of harmful circulating molecules and (2) replacement of protective plasma proteins consumed by the disease process [22].
Here, we hypothesize that TPE against plasma from healthy donors might (1) remove mediators of increased microvascular clotting (e.g., vWF with large and in many cases ultra-large vWF multimers) and (2) simultaneously compensate for the loss of factors important for regulating coagulation (e.g., activated protein C, ATIII) and fibrinolysis (e.g., ADAMTS13). In this study, we investigated in patients with early and severe septic shock the effect of a single TPE against FFPs on key factors regulating coagulation.

\section{Methods \\ Study population}

Data and bio-samples were acquired from both a recently completed prospective single-center non-randomized study [21] and a currently still-recruiting single-center randomized study (NCT03065751). We screened $n=1427$ patients submitted to our 14-bed medical ICU from July 2016 to March 2019 for the presence of sepsis per SEPSIS-3 definition [1] (Fig. 1). Twenty patients from the published feasibility trial and 11 patients from the subsequent ongoing randomized trial were included. All patients were treated according to the 2016 Surviving Sepsis Campaign (SSC) guidelines. The ethical committee of Hannover Medical School approved both study protocols, and written informed consent was obtained from participants or authorized representatives. The study was performed in accordance with the ethical standards laid down in the 1964 Declaration of Helsinki and its later amendments. Demographic and clinical data were obtained immediately before TPE.

\section{Inclusion and exclusion criteria}

Patients were included based on (i) septic shock with a need for vasopressors $<12 \mathrm{~h}$ prior to entry and (ii) profound systemic hypotension requiring norepinephrine (NE) doses of $>$ $0.4 \mu \mathrm{g} / \mathrm{kg} / \mathrm{min}$ despite adequate intravenous fluid resuscitation. All patients received a crystalloid fluid replacement with at least $30 \mathrm{~mL} / \mathrm{kg}$ body weight before TPE treatment. Fluid status was routinely assessed following a routine in-house standard operating procedure applying bedside transthoracic echocardiography (TTE) and measurement of inferior vena cava (IVC) diameter and/or stroke volume variation by pulse contour cardiac output (PiCCO) catheter. The median volume administered before TPE treatment was 3225 (22744479) $\mathrm{mL}$. As non-inclusion criteria, we defined unavailability of TPE within the first $6 \mathrm{~h}$ after study inclusion, pregnancy or breastfeeding, age < 18 years, end-stage chronic disease, and presence of a directive to withhold life-sustaining treatment.

\section{Therapeutic plasma exchange}

Vascular access was established by venous insertion of an 11-French two-lumen hemodialysis catheter. We used 
1427 patients screened between July 2016- March 2019

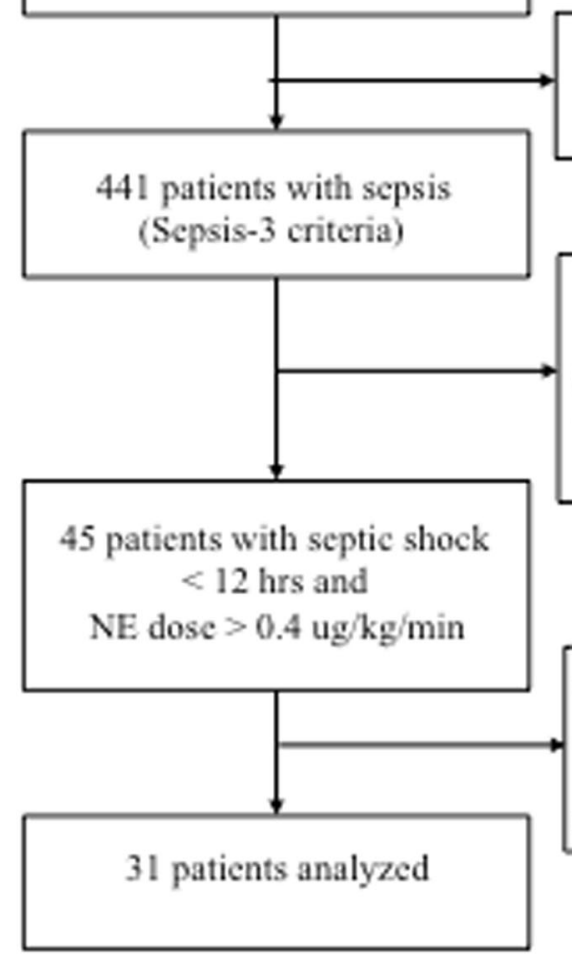

986 had a non-infectious condition requiring intenive care

164 patients had sepsis w/o shock 145 patients had shock $>12 \mathrm{hrs}$ 87 patients had NE dose $<0.4 \mathrm{ug} / \mathrm{kg} / \mathrm{min}$ 76 patients had shock $>12$ hrs $+N E<$ $0.4 \mathrm{ug} / \mathrm{kg} / \mathrm{min})$

4 patients had no blood collected before and after TPE

10 patients received no TPE (as part of randomization in the second study )

Fig. 1 Flow chart of study participants. NE, norepinephrine; TPE, therapeutic plasma exchange

one single TPE, as hemodynamic improvements were achieved only with the first TPE in a previous pilot study [23]. Centrifugal TPE was performed against a fixed dose of 12 units of FFP; therefore, total volumes exchanged varied between 1 and 1.5 times the plasma volume. Median blood flow was $60(55-63) \mathrm{mL} / \mathrm{min}$. Anticoagulation during TPE was achieved by regional citrate infusion. Post-filter calcium concentration was checked 15 min after starting the treatment as regulated by a local protocol at our institution. The citrate flow rate was adjusted to target post-filter ionized calcium concentrations of 0.5 to $0.6 \mathrm{mmol} / \mathrm{L}$. Additionally, systemic blood gas analysis was performed every 2 to $4 \mathrm{~h}$ to exclude electrolyte and acid-base dysbalances. Immediately before TPE treatment implementation, anti-allergic intravenous prophylaxis with ranitidine and clemastine was administered to all patients. In patients with acute kidney injury (AKI), hemodialysis was interrupted for the duration of TPE (110 (93-120) min). Blood samples were drawn before and after TPE. Patients were closely followed for the next 28 days, and survival was recorded. $\mathrm{NE}$ dose was titrated every $10-15 \mathrm{~min}$ to maintain a mean arterial pressure (MAP) above $65 \mathrm{mmHg}$.
Measurement of coagulation factors, ADAMTS13, vWF:Ag, and ULVWF

Patient plasma and serum blood samples were acquired before and after TPE. For blood sampling, $10 \mathrm{~mL}$ monovettes containing $1 \mathrm{~mL}$ of $0.13 \mathrm{~mol} / \mathrm{L}$ sodium citrate was used, and plasma was obtained by centrifugation divided into aliquots and stored at $-80^{\circ}$ until assayed. ADAMTS13 activity (ADAMTS13) was determined with the Technoclone ADAMTS13 activity ELISA kit. vWF antigen (vWF:Ag) was measured by an enzyme-linked immunosorbent assay [24]. vWF multimers were separated by gel electrophoresis $\left(60 \mathrm{~V}, 16^{\circ} \mathrm{C}, 15 \mathrm{~h}\right)$ using $1.7 \%$ and 1.3\% LGT agarose (Sigma-Aldrich, Seelze, Germany), detected by a vWF antibody conjugated with horseradish peroxidase (Dako, Hamburg, Germany) and visualized by an enhanced chemiluminescence (ECL detection kit; Amersham, Freiburg, Germany) [25]. The gels were recorded by a CCD camera and evaluated by the AphaView Q Software (Alpha Innotech).

Scores assessing coagulopathy were calculated, namely the sepsis-induced coagulopathy (SIC) and the International Society on Thrombosis and Haemostasis (ISTH) score for overt disseminated intravascular coagulation 
(DIC score). The SIC score was determined according to the suggested system by Iba et al. [26] with a score of $\geq$ 4 (out of max. 6) indicating sepsis-induced coagulopathy. The ISTH score was calculated as described previously [27], with a score of $\geq 5$ indicating overt DIC, and for Ddimer concentration, the following cutoff values were used: $0-0.5 \mathrm{mg} / \mathrm{L}=0$ points, $0.5-2 \mathrm{mg} / \mathrm{L}=2$ points, and $>2 \mathrm{mg} / \mathrm{L}=3$ points [28].

Clinical parameters were correlated with ADAMTS13 activity at the time of inclusion before TPE treatment was begun. For this purpose, patients with less severely reduced ADAMTS13 activity, e.g., $\geq 30 \%$, were compared to those with more profoundly reduced ADAMTS13 activity, e.g., $<30 \%$. The cutoff of $30 \%$ ADAMTS13 activity has been used before by Brunkhorst and coworkers [14].

\section{Statistical analysis}

Data were presented as median with interquartile range (IQR). Two-tailed $p$ values of less than 0.05 were considered to indicate statistical significance. Paired $t$ test or Wilcoxon test (as appropriate) was utilized in order to compare longitudinal values before (pre) and after (post) TPE. Unpaired $t$ test and Mann-Whitney test (for not normally distributed variables) were employed to compare unpaired values. For correlation analysis (ADAMTS13 activity to platelet count), non-linear regression analysis was performed and $R^{2}$ value was calculated accordingly. Survival data were analyzed by log-rank test and visualized by Kaplan-Meier curves. We used GraphPad Prism 7 (Graph Pad, La Jolla, CA, USA) and SPSS Statistics version 25 (SPSS Inc., Chicago, IL, USA) for data analysis and graph generation.

\section{Results}

\section{Cohort characterization}

Demographic and clinical details are summarized in Table 1. Seventy-one percent of the patients were men, and the median (IQR) age was $53(35-59)$ years. The lungs and the abdomen were the most common sites of infection. A causative pathogen was identified in $68 \%$ of the cases. All patients were treated with a combination of broad-spectrum antibiotics. Gram-negative and Gram-positive pathogens were the most commonly identified, and in $13 \%$ of patients, more than one pathogen was detected.

The median (IQR) SOFA score was 18 (16-20). Shock was profound with requiring about twice as high $\mathrm{NE}$ doses than required by the inclusion criteria. All patients displayed signs of severe hyper-inflammation as indicated by high levels of C-reactive protein (CRP) and procalcitonin (PCT). Ninety-four percent were mechanically ventilated and had an oxygenation index $\left(\mathrm{PaO}_{2} / \mathrm{FiO}_{2}\right)$ of 127 (92-195). AKI with a need for renal replacement therapy (RRT) was present in $68 \%$ of the patients at the time of inclusion.
Table 1 Demographic and clinical characteristics at baseline

\begin{tabular}{|c|c|}
\hline Category & Median (IQR)/n (\%) \\
\hline Age, years & $53(35-59)$ \\
\hline \multicolumn{2}{|l|}{ Sex, $n(\%)$} \\
\hline Male & $22(71)$ \\
\hline Female & $9(29)$ \\
\hline $\mathrm{BMI}, \mathrm{kg} / \mathrm{m}^{2}$ & $26(20.7-31.6)$ \\
\hline \multicolumn{2}{|l|}{ Sepsis onset, $n(\%)$} \\
\hline Ambulant & $19(61.3)$ \\
\hline Hospital & $12(38.7)$ \\
\hline \multicolumn{2}{|l|}{ Side of infection, $n$ (\%) } \\
\hline Pulmo & $17(54.8)$ \\
\hline Abdomen & $7(22.6)$ \\
\hline Urogenital & $1(3.2)$ \\
\hline Soft tissue & $4(12.9)$ \\
\hline Endocarditis & $1(3.2)$ \\
\hline More than one & $1(3.2)$ \\
\hline \multicolumn{2}{|l|}{ Identified pathogen, $n$ (\%) } \\
\hline Gram+ & $5(16.1)$ \\
\hline Gram- & $9(29)$ \\
\hline Fungi & $2(6.4)$ \\
\hline Viral & $1(3.2)$ \\
\hline More than one & $4(12.9)$ \\
\hline Non-identified & $10(32.3)$ \\
\hline SOFA & $18(16-20)$ \\
\hline Norepinephrine dose, $\mu \mathrm{g} / \mathrm{kg} / \mathrm{min}$ & $0.801(0.561-1.079)$ \\
\hline CRP, mg/L & $290(159-327)$ \\
\hline $\mathrm{PCT}, \mu \mathrm{g} / \mathrm{L}$ & $26(14-73)$ \\
\hline Mechanical ventilation, $n(\%)$ & $29(93.5)$ \\
\hline Oxygenation index $\left(\mathrm{PaO}_{2} / \mathrm{FiO}_{2}\right)$ & $127(92-195)$ \\
\hline Renal replacement therapy, $n(\%)$ & $21(67.7)$ \\
\hline
\end{tabular}

Given are the demographic and clinical characteristics at the time of study inclusion before TPE treatment. Values are presented as median (25 to $75 \%$ interquartile range) or if categorical as numbers and percentages $B M I$ body mass index, SOFA Sequential Organ Failure Assessment, CRP Creactive protein, $P C T$ procalcitonin

TPE was well tolerated, and no severe complications of TPE such as anaphylactoid reactions, citrate-induced hypocalcemia, catheter-related complications, and transfusionrelated lung injury were observed.

\section{Effect of TPE on routine coagulation parameters, antithrombin-III, protein C, and D-dimers}

While INR non-significantly decreased (1.6 (1.3-1.9) vs. 1.3 (1.2-1.7), $p=0.089)$, PTT remained unchanged following TPE (52 (40-76) s vs. $51(38-60) \mathrm{s}, p=0.084)$.

Fibrinogen was elevated before and slightly decreased after TPE to values considered in the normal range (4.1 $(2.8-7) \mathrm{g} / \mathrm{L}$ vs. $3.2(2.5-4.5) \mathrm{g} / \mathrm{L}, p<0.001$, Fig. 2a). As 


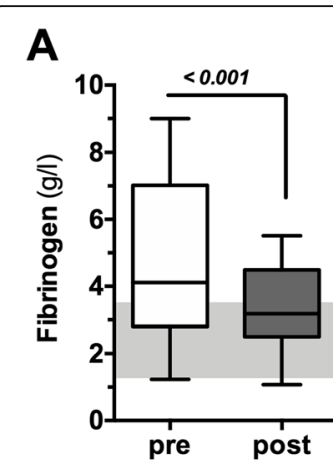

B

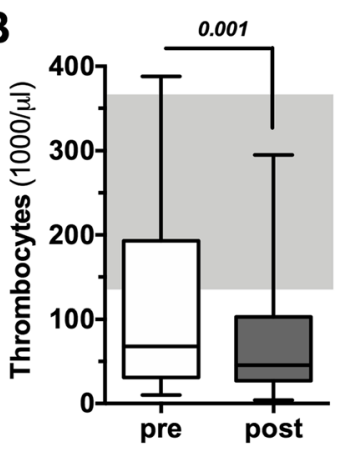

C

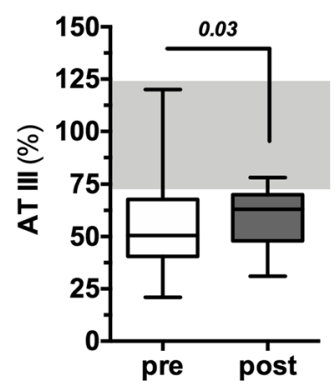

D
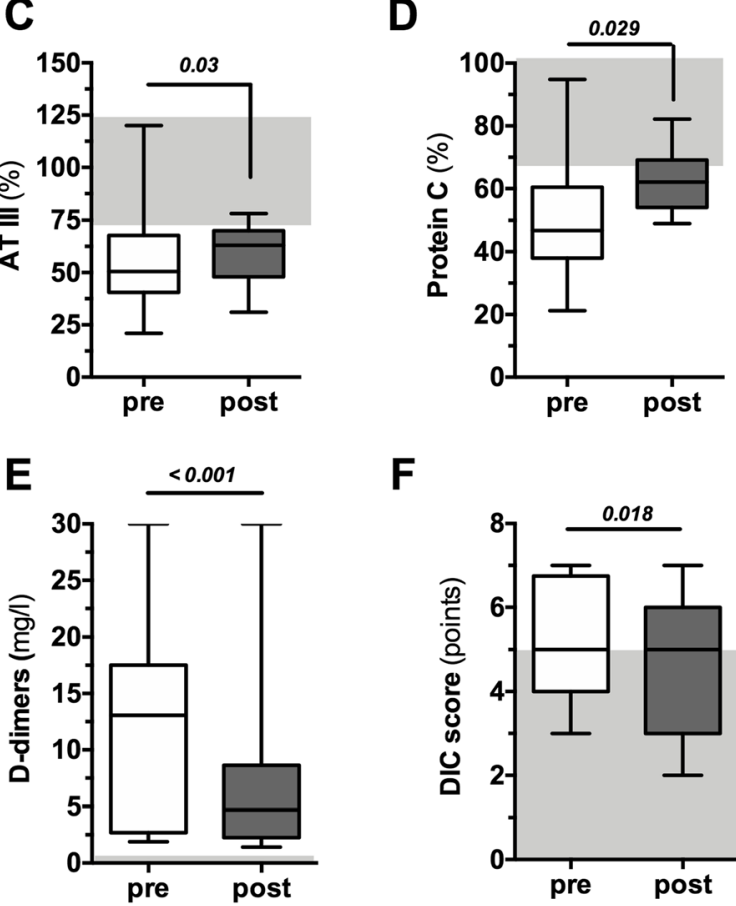

F

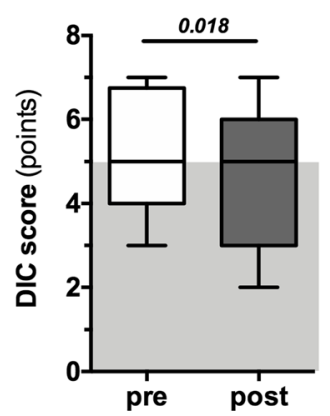

Fig. 2 Effect of TPE on routine coagulation parameters, antithrombin-III, protein C, and D-dimers. Box and whisker blots showing fibrinogen (a), platelet count (b), antithrombin-III activity (c), protein C activity (d), D-dimer concentration (e), and DIC score (f) immediately before and post (=after) TPE. Gray areas highlight the reference range for healthy individuals

an indicator of sepsis-associated coagulopathy, we observed a median SIC score at inclusion of 5 (4-6) and low platelet counts before TPE. Of note, platelets were even lower (68 (31-193) vs. 46 (27-103), $p=0.001$, Fig. 2b) after TPE.

Both antithrombotic acting proteins ATIII and protein $\mathrm{C}$ activity were markedly reduced before and significantly raised following TPE (ATIII, 51 (41-61) \% vs. 63 (48-70) \%, $p=0.029$, Fig. 2c; protein C, 47 (38-60) \% vs. $62(54-69) \%, p=0.029$, Fig. 2d). D-dimers were severely elevated in all patients and significantly declined following TPE (13.1 (2.7-17.5) mg/L vs. 4.7 (2.3-8.6) $\mathrm{mg} / \mathrm{L}$, $p<0.001$, Fig. 2e). In a subset of 20 patients, the DIC score could be determined. The DIC score significantly

decreased following TPE $(5.1 \pm 1.5$ points vs. $4.5 \pm 1.5$ points, $p=0.018$, Fig. 2f).

\section{Effect of TPE on ADAMTS13, vWF:Ag, and vWF:Ag/ ADAMTS-13 ratio}

ADAMTS13 was reduced below normal limits of 50\% before TPE in 29/31 (94\%) patients. Moreover, a subset of $17 / 31$ (55\%) patients showed severely reduced ADAMTS13 activity below 30\%. ADAMTS13 increased from $27(21-42) \%$ before to $47(38-62) \%$ after TPE $(p<0.001$, Fig. 3a).

In contrast, vWF:Ag was increased at study inclusion and reduced by TPE (353 (206-492) IU/dL vs. 170 (117-232) IU/dL, $p<0.001$, Fig. 3b).

Consequentially, the elevated ratio of vWF:Ag to ADAMTS13 at inclusion was significantly reduced by TPE (12.3 (7.6-18.5) vs. 3.7 (2.3-4.6), $p<0.001$, Fig. 3c).

ULWF were detectable in a significant amount before TPE in $7 / 31$ (23\%) patients and were improved in 5 (71\%) and completely normalized in 2 of them after TPE (data not shown).

\section{ADAMTS13 and correlation with clinical parameters of disease severity at inclusion}

Patients with a profound reduction of ADAMTS13 (defined by an activity <30\%) were compared to patients with less severe suppression of ADAMTS13 activity ( $\geq$ $30 \%)$ in terms of clinical parameters at the time of inclusion before TPE treatment. Patients with ADAMTS13 activity $\geq 30 \%$ showed a trend to less organ dysfunction as indicated by lower SOFA scores (16 (14-19) vs. 19 (17-22), $p=0.095$, Fig. 4a) and comparable severity of hemodynamic shock (NE dose, 0.884 (0.639-1.158) $\mu \mathrm{g} /$ $\mathrm{kg} / \mathrm{min}$ vs. $0.667(0.516-1.273) \mu \mathrm{g} / \mathrm{kg} / \mathrm{min}, \quad p=0.529$, Fig. 4b). However, the median DIC score was higher in patients with ADAMTS13 activity <30\% (6.5 (5-7) points vs. 4 (3-5.5) points, $p=0.002$, Fig. $4 c)$.

Median LDH was 496 (336-1375) U/L in patients with ADAMTS13 < 30\%, compared to 295 (212-839) U/L if the activity was $\geq 30 \%$ ( $p=0.178$, Fig. $4 \mathrm{~d}$ ). Platelets were severely reduced in patients with ADAMTS13 < 30\% (39 $(26-63) \times 1000 / \mu \mathrm{L}$ and only slightly reduced in those with ADAMTS13 $\geq 30 \%(193(86-281) \times 1000 / \mu \mathrm{L}, p=0.004$, Fig. 4e). Non-linear regression yielded a significant correlation between ADAMTS13 activity and platelet count $\left(p=0.001, R^{2}=0.316\right.$, Fig. 4f $)$.

\section{ADAMTS13, vWF:Ag, and survival}

The overall 30-day survival was $42 \%$ (13/31 patients), and the mean time from termination of TPE treatment to death was $8.4 \pm 8.2$ days. Survival was $54 \%(7 / 13)$ in patients with ADAMTS13 $\geq 30 \%$ and $35 \%(6 / 17)$ in patients with ADAMTS13 $<30 \%$ measured at inclusion before performing TPE treatment. The hazard ratio (HR) 

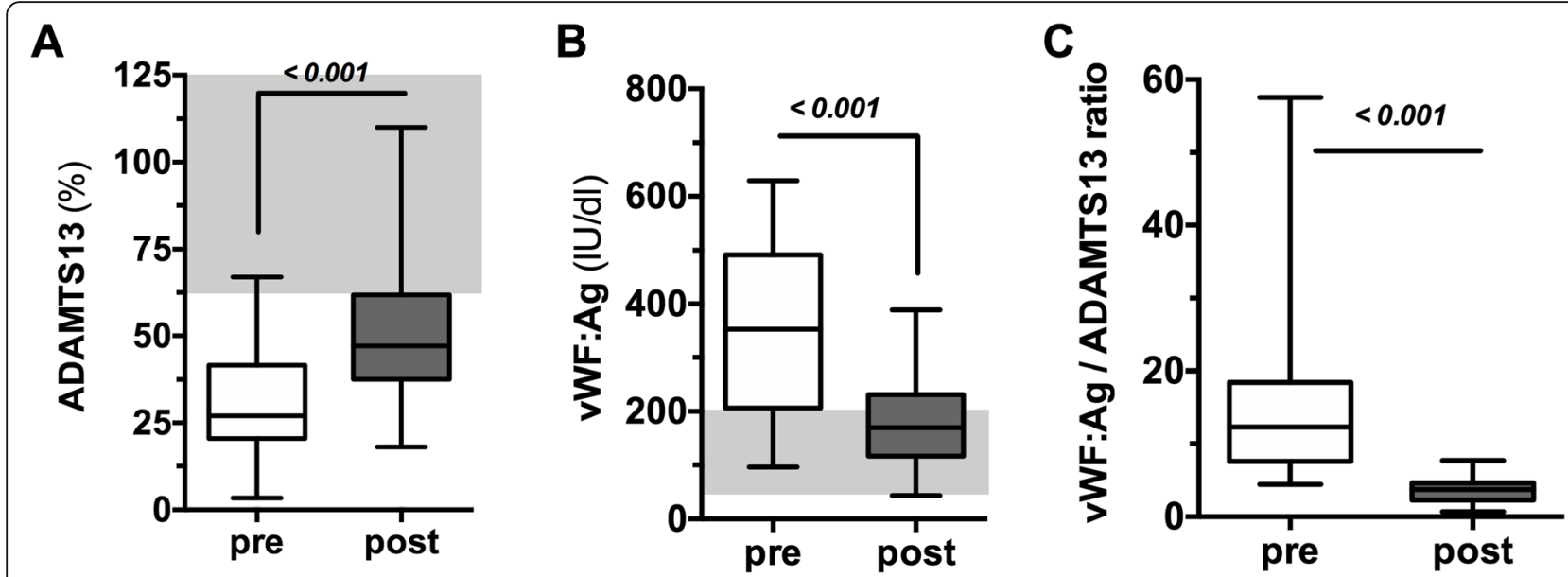

Fig. 3 Effect of TPE on ADAMTS13, VWF:Ag, and VWF:Ag/ADAMTS-13 ratio. Box and whisker blots showing ADAMTS13 activity (a), VWF:Ag (b), and VWF:Ag/ADAMTS13 ratio (c) immediately before start and post (=after) TPE. Gray areas highlight the reference range for healthy individuals

was 1.46 (95\% CI $0.56-3.79, p=0.45$, Suppl. Figure 1). ADAMTS13 and vWF:Ag were comparable between surviving and non-surviving patients, both at inclusion and after TPE treatment (Suppl. Figure 2A and C). ADAMTS13 activity was raised by TPE by $82(50-117)$ $\%$ in survivors and by $72(16-126) \%$ in non-survivors $(p=0.589$, Suppl. Figure 2B). Contrastively, vWF:Ag was reduced by TPE by $54 \%$ (41-64) in surviving patients and by $47 \%(37-67)$ in non-surviving patients $(p=0.923$, Suppl. Figure 2D).

In terms of shock reversal, we defined subgroups that demonstrated (1) any reduction of NE and (2) a more marked NE reduction by more than $20 \%$ of baseline NE dose following TPE treatment (Suppl. Figure 2E and F). TPE-responsive patients with regard to hemodynamic stabilization showed an increase of ADAMTS13 by 83 (35-128) \% while patients without any NE dose reduction by $60 \%(20-104)(p=0.482$, Suppl. Figure 2E). ADAMTS13 activity changed by $86(38-128) \%$ in patients with a NE dose reduction of greater than $20 \%$ and by $60 \%(27-146)$ in patients with NE dose reduction of $\leq 20 \%(p=0.513$, Suppl. Figure 2F).

\section{Discussion}

This prospective single-center explorative study examined the effects of early TPE on endothelial activation and coagulation-related parameters in septic shock. Patients included in this investigation experienced a severe form of septic shock as indicated by exceedingly high NE requirement, inflammatory markers, and a high prevalence of multi-organ failure. Low platelet counts with high DIC scores indicated the possibility of overt DIC in these patients. However, traditional DIC scores like the ISTH-DIC Score have been reported to have a poor sensitivity, especially in infectious diseases [29]. The SIC score has been suggested recently to be more specific in sepsis [26] but has been questioned in a validation cohort, as the ISTH score had greater power than the SIC score in predicting ICU mortality in septic patients [30]. We acknowledge the limitation of clinical scores to adequately comprehend DIC in septic patients. In this report, we have followed the current expert opinions that recommend to use the SIC score as a screening tool to identify patients with possible sepsis-associated coagulopathy and subsequentially calculate an overt DIC score like the ISTH score [29]. At the same time, hyper-fibrinolysis as indicated by elevated Ddimer concentrations was present. Patients with septic shock displayed a significant shift of the hemostatic balance, characterized by activation of pro-coagulant proteins and attenuation of anti-coagulant activity at the same time. In this sense, pro-coagulatory factors such as vWFAg, ULVWF, and fibrinogen were markedly increased at inclusion while anti-coagulant proteins such as ATIII, protein $\mathrm{C}$, and ADAMTS13 were depleted. These observations are well in line with two previous investigations describing these changes in septic patients $[14,20]$. Especially, the vWF-cleaving protease ADAMTS13 was reduced in $94 \%$ and severely reduced in $55 \%$ of patients. This deficiency of ADAMTS13 was associated with both high vWF:Ag and ULVWF concentrations as well as with thrombocytopenia and increased LDH concentrations. Both changes are characteristic for the presence of thrombotic microangiopathy (TMA) with ongoing hemolysis as a consequence of low ADAMTS13 activity. These cardinal features of TMA by a relative deficiency in ADAMTS13 activity were described previously in septic patients and were associated with worse outcomes [13, 17, 19].

In this analysis, we observed higher DIC scores in patients with low ADAMTS13. In contrast, an earlier study by Peigne et al. found no correlation between the DIC score and ADAMTS13 [18]. A possible explanation concerning these contradictory results might be the 

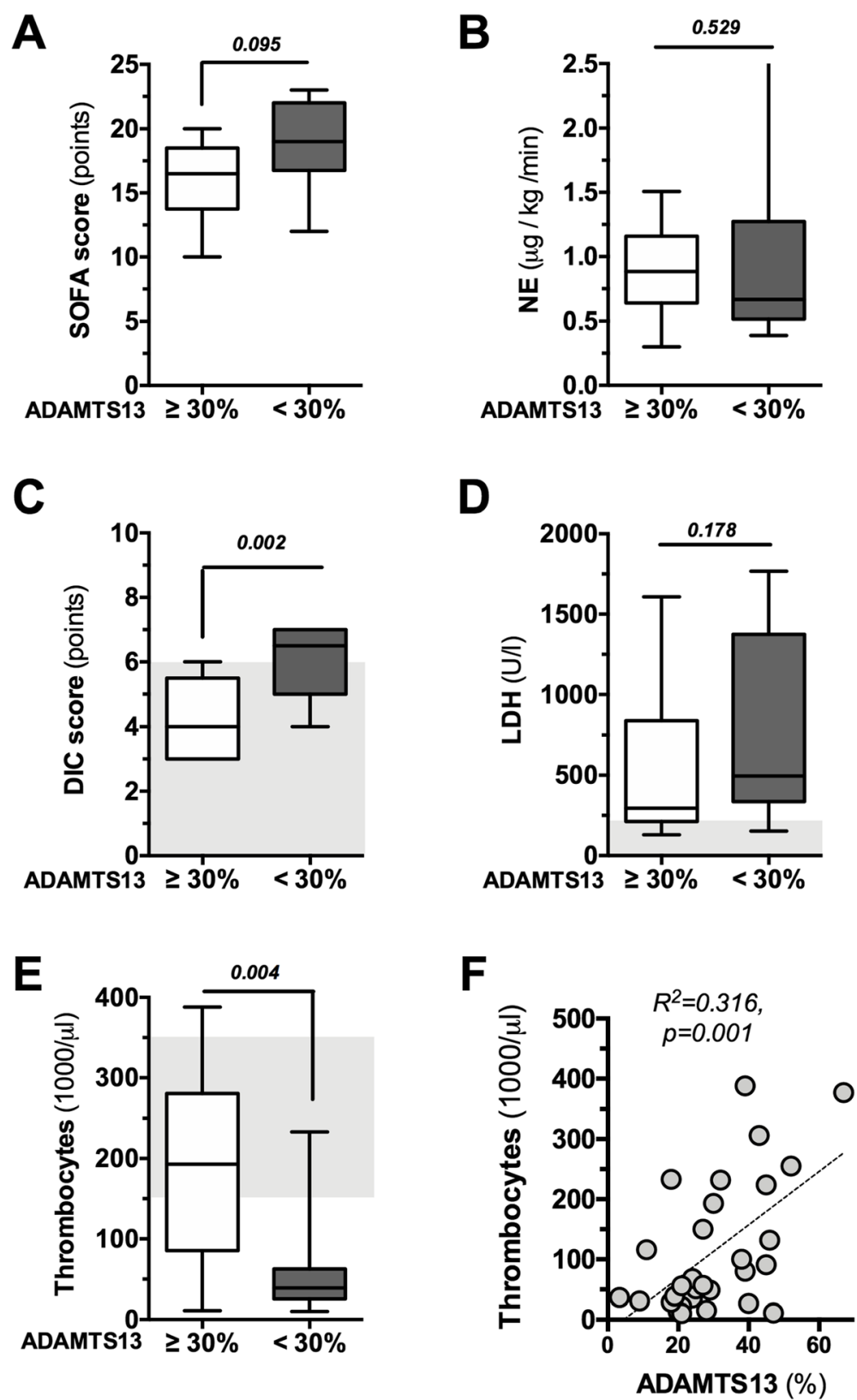

Fig. 4 Correlation of ADAMTS13 with clinical parameters of disease severity. Box and whisker blots demonstrating SOFA scores (a), NE dose (b), DIC score $(\mathbf{c})$, LDH $(\mathbf{d})$, and platelet count (e) for both patients with profound reduction of ADAMTS13 activity $(<30 \%)$ and patients with less severe suppression of ADAMTS13 activity $(\geq 30 \%)$ at the time of inclusion before TPE treatment. $\mathbf{f}$ Non-linear regression of ADAMTS13 activity to platelet count

differences in the severity and duration of septic shock as well as the severity of organ failures between these two cohorts. Peigne et al. described the lower need for both mechanical ventilation and renal replacement therapy. Additionally, inflammatory activity indicated by IL6 concentration was more than ten times higher in our cohort with a median (IQR) IL-6 of $10,810 \mathrm{pg} / \mathrm{mL}$ $(2538-27,570)$ compared to $895 \mathrm{pg} / \mathrm{mL}(330-1843)$ in the study by Peigne et al.
The clinical relevance of reduced ADAMTS13 is well known from a disease termed TTP in which the formation of ULVWF multimers due to reduced ADAMTS13 leads to thrombocytopenia, hemolysis, AKI, and (often severe) neurological symptoms. Supplementation of ADAMTS13-that is found in high concentration in the plasma from healthy donors-by TPE can (in some cases within minutes) positively affect these symptoms. 
The question arises if it is possible to influence the slightly more complex dysbalance of the hemostatic system in clinical sepsis scenario. Treatment with activated protein $\mathrm{C}$ (APC) has demonstrated efficacy in selected patients, e.g., with purpura fulminans $[7,8]$, but has failed in larger more general sepsis populations [10]. No recombinant ADAMTS13 is available until now, and regular renal replacement regimens have no ability to reduce pro-coagulant factors. We hypothesized that the exchange of septic shock plasma with fresh frozen plasma (FFP) from healthy donors does not only lead to a reduction of harmful proteins but also rather replenishes protective factors that had been consumed by sepsis analogously to TTP. In fact, a key finding of our analysis is that TPE might have the potential to partially attenuate this dysbalance by removing pro- and simultaneously replacing anti-coagulant factors.

A high vWF:Ag/ADAMTS13 ratio has been associated previously with the development of TMA, higher severity of shock, degree of organ failure, and worse outcomes in patients with septic shock [20]. Here, we not only can confirm the elevation of this ratio in septic shock but we can also demonstrate a putative therapeutic strategy to recalibrate this disequilibrium in the coagulation process.

This study has important limitations, mainly its small sample size affecting the power of the results, the singlecenter setting, and the lack of a clinical correlate that reflects the actual microcirculatory state. Currently available methods in clinical use for microvascular perfusion monitoring such as biomarkers of endothelial damage/ activation biomarkers), videomicroscopic techniques, tissue oxygenation evaluation techniques, and partial pressure of carbon dioxide $\left(\mathrm{pCO}_{2}\right)$-based evaluation techniques were not employed in this study. Unfortunately, the size of this study was too small to demonstrate significant differences in ADAMTS13, vWF:Ag, or the presence of ULVWF between survivors and nonsurvivors or patients with and without shock reversal by TPE, respectively. As this study was a single-center study, there is a potential for patient selection bias. In addition, the intervention was administered at a fixed dose, which precludes us from providing data on the effects at different dosages or time frames.

\section{Conclusions}

In this prospective study, septic shock was associated with activation of pro-coagulant pathways such as vWF: $\mathrm{Ag}$ and ultra-large VWF multimers and consumption of anti-coagulant factors such as ATIII, protein $C$, and ADAMTS13 activities. Early TPE treatment partially attenuated this dysbalance by removing pro- and replacing anti-coagulant factors. It has yet to be determined by an appropriately powered RCT if TPE might improve the outcome in septic patients.

\section{Supplementary information}

Supplementary information accompanies this paper at https://doi.org/10. 1186/s13054-020-2799-5.

Additional file 1: Figure S1. Thirty-day survival. Kaplan Meier graphs showing the 30-day survival course in (A) the overall cohort (41.9\% survival (13/31 patients)) as well as (B) in patients with ADAMTS13 $\geq 30 \%$ (53.9\% survival (7/13)) and (C) in patients with ADAMTS13 < 30\% $(35.3 \%$ survival (6/17)). ADAMTS13 activity was measured at inclusion before performing TPE treatment.

Additional file 2: Figure S2. ADAMTS13 and $\mathrm{WWF:Ag}$ and clinical outcome. Box and whisker blots demonstrating ADAMTS13 activity (A) and VWF:Ag (C) pre- and post TPE as well as \% change of ADAMTS13 activity (B) and VWF:Ag (D) by TPE. Percentage change of ADAMTS13 activity is also displayed dependent on achieved reduction of NE (E) dose and reduction of NE dose by $>20 \%$ (F) following TPE.

\begin{abstract}
Abbreviations
ADAMTS13: A disintegrin and metalloprotease with a thrombospondin type 1 motif, member 13; AKI: Acute kidney injury; BMI: Body mass index; CRP: Creactive protein; FFP: Fresh frozen plasma; MAP: Mean arterial pressure; NE: Norepinephrine; PCT: Procalcitonin; RCT: Randomized controlled trial; RRT: Renal replacement therapy; SOFA: Sequential Organ Failure Assessment; TPE: Therapeutic plasma exchange; ULWWF: Ultra-large von Willebrand factor multimers; VWF: von Willebrand factor; vWF:Ag: von Willebrand factor antigen
\end{abstract}

Authors' contributions

KS collected the clinical data from the PDMS and generated the figures for publication. BMWS was the leading nephrology consultant coordinating and performing the plasma exchange on our unit. UB performed the laboratory experiments measuring the ADAMTS13, VWF:Ag, and ULWWF. JJS and BS recruited the patients. $\mathrm{KS}, \mathrm{UB}, \mathrm{CB}, \mathrm{SD}$, and $\mathrm{MMH}$ interpreted the data. $\mathrm{KS}$ and SD wrote the manuscript and calculated the statistics. SD had the original idea for both trials and wrote the proposals. All authors read and approved the final manuscript.

\section{Funding}

No funding was received.

\section{Availability of data and materials}

The datasets used and analyzed during the current study are available from the corresponding author on reasonable request.

\section{Ethics approval and consent to participate}

The ethical committee of Hannover Medical School approved the protocols of both studies (no. 2786-2015 and ClinicalTrials.gov NCT03065751), and written informed consent was obtained from participants or authorized representatives. The study was performed in accordance with the ethical standards laid down in the 1964 Declaration of Helsinki and its later amendments.

\section{Consent for publication}

Not applicable

\section{Competing interests}

The authors declare that they have no competing interests.

\section{Author details}

${ }^{1}$ Department of Gastroenterology, Hepatology and Endocrinology, Hannover Medical School, Hannover, Germany. ${ }^{2}$ Department of Nephrology and Hypertension, Hannover Medical School, Carl-Neuberg-Str.1, 30625 Hannover, Germany. ${ }^{3}$ Department of Respiratory Medicine and German Centre of Lung Research (DZL), Hannover Medical School, Hannover, Germany. ${ }^{4}$ Medilys Laborgesellschaft mbH, Hamburg, Germany. ${ }^{5}$ Department of Anaesthesiology and Critical Care, University Medicine Bonn, Bonn, Germany. 
Received: 10 December 2019 Accepted: 18 February 2020

Published online: 02 March 2020

\section{References}

1. Singer M, Deutschman CS, Seymour CW, Shankar-Hari M, Annane D, Bauer $M$, et al. The Third International Consensus Definitions for Sepsis and Septic Shock (Sepsis-3). Jama. 2016;315(8):801-10.

2. Engel C, Brunkhorst FM, Bone HG, Brunkhorst R, Gerlach H, Grond S, et al. Epidemiology of sepsis in Germany: results from a national prospective multicenter study. Intensive Care Med. 2007;33(4):606-18.

3. Angus DC, van der Poll T. Severe sepsis and septic shock. N Engl J Med. 2013;369(21):2063.

4. Aird WC. The role of the endothelium in severe sepsis and multiple organ dysfunction syndrome. Blood. 2003;101(10):3765-77.

5. Powars D, Larsen R, Johnson J, Hulbert T, Sun T, Patch MJ, et al. Epidemic meningococcemia and purpura fulminans with induced protein $C$ deficiency. Clin Infect Dis. 1993;17(2):254-61.

6. Lorente JA, Garcia-Frade LJ, Landin L, de Pablo R, Torrado C, Renes E, et al. Time course of hemostatic abnormalities in sepsis and its relation to outcome. Chest. 1993:103(5):1536-42.

7. White B, Livingstone W, Murphy C, Hodgson A, Rafferty M, Smith OP. An open-label study of the role of adjuvant hemostatic support with protein C replacement therapy in purpura fulminans-associated meningococcemia. Blood. 2000;96(12):3719-24.

8. Knoebl P, Schellongowski P, Staudinger T, Sperr WR, Scheibenpflug C. Treatment of infection-associated purpura fulminans with protein $\mathrm{C}$ zymogen is associated with a high survival rate. Blood. 2013;122(21):3606.

9. Bernard GR, Vincent JL, Laterre PF, LaRosa SP, Dhainaut JF, Lopez-Rodriguez $A$, et al. Efficacy and safety of recombinant human activated protein $C$ for severe sepsis. N Engl J Med. 2001;344(10):699-709.

10. Ranieri VM, Thompson BT, Barie PS, Dhainaut JF, Douglas IS, Finfer $S$, et al. Drotrecogin alfa (activated) in adults with septic shock. N Engl J Med. 2012; 366(22):2055-64

11. Crawley JT, de Groot R, Xiang Y, Luken BM, Lane DA. Unraveling the scissile bond: how ADAMTS13 recognizes and cleaves von Willebrand factor. Blood. 2011;118(12):3212-21.

12. Levy GG, Nichols WC, Lian EC, Foroud T, McClintick JN, McGee BM, et al. Mutations in a member of the ADAMTS gene family cause thrombotic thrombocytopenic purpura. Nature. 2001:413(6855):488-94.

13. Aibar J, Castro P, Espinosa G, Fernandez S, Hernandez C, Rinaudo M, et al. ADAMTS-13 in critically ill patients with septic syndromes and noninfectious systemic inflammatory response syndrome. Shock. 2015;43(6):556-62.

14. Bockmeyer CL, Claus RA, Budde U, Kentouche K, Schneppenheim R, Losche W, et al. Inflammation-associated ADAMTS13 deficiency promotes formation of ultra-large von Willebrand factor. Haematologica. 2008;93(1):137-40.

15. Kremer Hovinga JA, Zeerleder S, Kessler P, Romani de Wit T, van Mourik JA, Hack CE, et al. ADAMTS-13, von Willebrand factor and related parameters in severe sepsis and septic shock. J Thromb Haemost. 2007:5(1 1):2284-90.

16. Stockschlaeder M, Schneppenheim R, Budde U. Update on von Willebrand factor multimers: focus on high-molecular-weight multimers and their role in hemostasis. Blood Coagulation Fibrinolysis. 2014;25(3):206-16.

17. Ono T, Mimuro J, Madoiwa S, Soejima K, Kashiwakura Y, Ishiwata A, et al. Severe secondary deficiency of von Willebrand factor-cleaving protease (ADAMTS13) in patients with sepsis-induced disseminated intravascular coagulation: its correlation with development of renal failure. Blood. 2006; 107(2):528-34.

18. Peigne V, Azoulay E, Coquet I, Mariotte E, Darmon M, Legendre P, et al. The prognostic value of ADAMTS13 (a disintegrin and metalloprotease with thrombospondin type 1 repeats, member 13) deficiency in septic shock patients involves interleukin-6 and is not dependent on disseminated intravascular coagulation. Crit Care. 2013;17(6):R273.

19. Lin JJ, Chan OW, Hsiao HJ, Wang Y, Hsia SH, Chiu CH. Decreased ADAMTS 13 activity is associated with disease severity and outcome in pediatric severe sepsis. Medicine (Baltimore). 2016;95(16):e3374.

20. Claus RA, Bockmeyer CL, Budde U, Kentouche K, Sossdorf M, Hilberg T, et al. Variations in the ratio between von Willebrand factor and its cleaving protease during systemic inflammation and association with severity and prognosis of organ failure. Thromb Haemost. 2009;101(2):239-47.

21. Knaup H, Stahl K, Schmidt BMW, Idowu TO, Busch M, Wiesner O, et al. Early therapeutic plasma exchange in septic shock: a prospective open-label nonrandomized pilot study focusing on safety, hemodynamics, vascular barrier function, and biologic markers. Crit Care. 2018:22(1):285.

22. Sloan SR, Andrzejewski C Jr, Aqui NA, Kiss JE, Krause PJ, Park YA. Role of therapeutic apheresis in infectious and inflammatory diseases: current knowledge and unanswered questions. J Clin Apher. 2015;30(5):259-64.

23. David S, Hoeper MM, Kielstein JT. Plasma exchange in treatment refractory septic shock: presentation of a therapeutic add-on strategy. Medizinische Klinik, Intensivmedizin und Notfallmedizin. 2017;112(1):42-6.

24. Cejka J. Enzyme immunoassay for factor VIII-related antigen. Clin Chem. 1982;28(6):1356-8.

25. Budde U, Schneppenheim R, Eikenboom J, Goodeve A, Will K, Drewke E, et al. Detailed von Willebrand factor multimer analysis in patients with von Willebrand disease in the European study, molecular and clinical markers for the diagnosis and management of type 1 von Willebrand disease (MCMDM-1WWD). J Thromb Haemost. 2008;6(5):762-71.

26. Iba T, Nisio MD, Levy JH, Kitamura N, Thachil J. New criteria for sepsisinduced coagulopathy (SIC) following the revised sepsis definition: a retrospective analysis of a nationwide survey. BMJ Open. 2017;7(9):e017046.

27. Taylor FB Jr, Toh CH, Hoots WK, Wada H, Levi M, Scientific Subcommittee on Disseminated Intravascular Coagulation of the International Society on $\mathrm{T}$ et al. Towards definition, clinical and laboratory criteria, and a scoring system for disseminated intravascular coagulation. Thromb Haemost. 2001; 86(5):1327-30

28. Gando S, Iba T, Eguchi Y, Ohtomo Y, Okamoto K, Koseki K, et al. A multicenter, prospective validation of disseminated intravascular coagulation diagnostic criteria for critically ill patients: comparing current criteria. Crit Care Med. 2006;34(3):625-31.

29. Iba T, Levy JH, Warkentin TE, Thachil J, van der Poll T, Levi M, et al. Diagnosis and management of sepsis-induced coagulopathy and disseminated intravascular coagulation. J Thromb Haemost. 2019;17(11): 1989-94.

30. Ding R, Wang Z, Lin Y, Liu B, Zhang Z, Ma X. Comparison of a new criteria for sepsis-induced coagulopathy and International Society on Thrombosis and Haemostasis disseminated intravascular coagulation score in critically ill patients with sepsis 3.0: a retrospective study. Blood Coagul Fibrinolysis. 2018;29(6):551-8.

\section{Publisher's Note}

Springer Nature remains neutral with regard to jurisdictional claims in published maps and institutional affiliations.

Ready to submit your research? Choose BMC and benefit from

- fast, convenient online submission

- thorough peer review by experienced researchers in your field

- rapid publication on acceptance

- support for research data, including large and complex data types

- gold Open Access which fosters wider collaboration and increased citations

- maximum visibility for your research: over $100 \mathrm{M}$ website views per year

At $\mathrm{BMC}$, research is always in progress.

Learn more biomedcentral.com/submission 